\title{
Intensive blood pressure treatment in coronary artery disease: implications from the Systolic Blood Pressure Intervention Trial (SPRINT)
}

\author{
Jiabin Zang ${ }^{1,2,3} \cdot$ Jianwen Liang ${ }^{2,3} \cdot$ Xiaodong Zhuang $\mathbb{C}^{4,5} \cdot$ Shaozhao Zhang ${ }^{4,5} \cdot$ Xinxue Liao $\mathbb{1}^{4,5} \cdot$ Guifu Wu $\mathbb{C}^{2,3,5}$
}

Received: 14 August 2020 / Revised: 2 January 2021 / Accepted: 20 January 2021 / Published online: 15 February 2021

(c) The Author(s) 2021. This article is published with open access

\begin{abstract}
To investigate the optimal blood pressure (BP) in patients with coronary artery disease (CAD), we conducted subgroup analysis using SPRINT data. The study sample included 1206 participants with CAD (of whom 692 underwent coronary revascularization) and 8127 participants without CAD. Participants were randomized into two groups (systolic BP target of $140 \mathrm{~mm} \mathrm{Hg}$ vs. $120 \mathrm{~mm} \mathrm{Hg}$ ). The primary outcome was a composite of cardiovascular events. After a median follow-up of 3.9 years, the hazard ratios (HRs) for the primary outcome were 0.65 (95\% confidence interval (CI) $0.53-0.79)$ and 1.05 (95\% CI 0.76-1.46) among those in the non-CAD and CAD subgroups, respectively ( $P$ value for interaction 0.02 ). Intensive BP treatment was a protective factor for all-cause death (HR 0.60,95\% CI 0.37-0.96) in the CAD subgroup, compared with standard BP treatment. The HRs $(95 \%$ CI ) for stroke were $3.57(1.17-10.85)$ and $1.03(0.29-3.62)$ among those in the coronary revascularization and non-revascularization subgroups, respectively ( $P$ value for interaction 0.13$)$. For safety events, intensive BP treatment increased the risk of hypotension (HR 2.00, 95\% CI 1.06-3.79) and electrolyte abnormalities (HR 2.38, 95\% CI 1.25-4.56) in the CAD subgroup, while the risk of serious adverse events did not increase (HR 1.03, 95\% CI $0.88-1.20$ ). These results suggest that positive benefits from intensive BP treatment might be attenuated in patients with $\mathrm{CAD}$ who are under better secondary prevention. The risk of stroke might increase at the systolic BP target of $120 \mathrm{~mm} \mathrm{Hg}$ in case of coronary revascularization, although the confidence interval was wide.
\end{abstract}

These authors contributed equally: Jiabin Zang, Jianwen Liang

Supplementary information The online version contains supplementary material available at https://doi.org/10.1038/s41371021-00494-8.

Xinxue Liao

liaoxinx@mail.sysu.edu.cn

$\triangle$ Guifu Wu

wuguifu@mail.sysu.edu.cn

1 Department of Cardiology, The Third Affiliated Hospital of Sun Yat-sen University, Guangzhou, Guangdong, China

2 Department of Cardiology, The Eighth Affiliated Hospital of Sun Yat-sen University, Shenzhen, Guangdong, China

3 Guangdong Innovative Engineering and Technology Research Center for Assisted Circulation, Shenzhen, Guangdong, China

4 Department of Cardiology, The First Affiliated Hospital of Sun Yat-sen University, Guangzhou, Guangdong, China

5 NHC Key Laboratory on Assisted Circulation, Sun Yat-sen University, Guangzhou, Guangdong, China

\section{Introduction}

Large-scale prospective studies have demonstrated that elevated blood pressure (BP) is associated with coronary artery disease (CAD) [1-4]. The prevalence of hypertension ranges from 30 to $70 \%$ in individuals with pre-existing CAD [5], and a previous study demonstrated that a $20 \mathrm{~mm}$ $\mathrm{Hg}$ rise in systolic blood pressure (SBP) or a $10 \mathrm{~mm} \mathrm{Hg}$ rise in diastolic blood pressure (DBP) results in a twofold increase in the risk of mortality among patients with ischemic heart disease aged 40-69 years [6]. Meanwhile, a reduction in SBP of $5 \mathrm{~mm} \mathrm{Hg}$ can decrease the risk of death from cardiovascular disease (CVD) by 9\% [7]. Currently, few clinical trials are primarily designed to evaluate optimal BP targets in patients with CAD. Therefore, there are no standardized BP targets for patients with $\mathrm{CAD}$, and current clinical practice is largely based on expert consensus with scant clinical trial evidence. Recent practice guidelines recommend a BP target of less than $130 / 80 \mathrm{~mm} \mathrm{Hg}$ in individuals with stable ischemic heart disease (SIHD), although it was acknowledged that this recommendation 
was supported by limited data [8]. Nevertheless, evidence suggests that adopting a "lower is better" approach for BP is far from ideal and that the BP targets vary depending on patient characteristics [9-12].

In hypertensive patients with $\mathrm{CAD}$, atherosclerotic lesions and arterial stiffness tend to be more severe, resulting in a lower DBP and increased pulse pressure. Patients who have undergone coronary revascularization seem to be more tolerant of lower DBP than those who had not, which may be partly explained by improved myocardial perfusion [13]. When performed using the proper revascularization strategy for appropriate patients, coronary revascularization can offer survival benefits in CAD; however, there is a need for further research to define the optimal BP target and therapeutic benefit of intensive BP treatment in this population.

The Systolic Blood Pressure Intervention Trial (SPRINT) examined the effect of intensive BP treatment (SBP target $<120 \mathrm{~mm} \mathrm{Hg}$ ) in hypertensive patients with high cardiovascular risk [14]. Using data from the SPRINT trial, we conducted a post-hoc analysis to investigate the optimal BP in CAD populations and in a subset of CAD patients who had undergone revascularization.

\section{Methods}

\section{Data source and study population}

Data were collected from the SPRINT study, which is a National Heart, Lung, and Blood Institute (NHLBI)-sponsored trial. SPRINT was a randomized, controlled, multicenter open-label trial that evaluated the effects of standard (SBP target $<140 \mathrm{~mm} \mathrm{Hg}$ ) versus intensive (SBP target $<120$ $\mathrm{mm} \mathrm{Hg}$ ) BP treatment among 9361 adults with hypertension (SBP of $130-180 \mathrm{~mm} \mathrm{Hg}$ ). Patients with diabetes mellitus, prior stroke, congestive heart failure, and advanced chronic kidney disease were excluded. In this study, patients diagnosed with CAD by a physician were randomized to either a standard or intensive group. Patients were followed up monthly for the first 3 months and every 3 months thereafter until 5 years or closeout. Coronary revascularization was defined by self-report of a history of percutaneous coronary intervention or coronary artery bypass grafting. The detailed inclusion and exclusion criteria are presented in the SPRINT study [14].

\section{Interventions and measurements}

BP measurements were taken with the participants in a seated position using the same automatic device in an unattended office. The mean of three measurements was included in the analysis. During a median follow-up of
3.9 years, BP-lowering medications were adjusted to an SBP target of $135-139 \mathrm{~mm} \mathrm{Hg}$ in the standard group and to less than $120 \mathrm{~mm} \mathrm{Hg}$ in the intensive group.

\section{Clinical outcomes and safety events}

Composite events of myocardial infarction, acute coronary syndromes, heart failure, stroke, and cardiovascular death were defined as the primary outcomes. Secondary outcomes included all-cause death and the various elements of the composite primary outcome.

In this study, the following conditions were recorded as safety events: hypotension, syncope, electrolyte abnormality, injurious fall, acute kidney injury, and bradycardia. Serious adverse events (SAEs) were defined as fatal events that first caused significant dysfunction and required medical intervention or hospitalization.

\section{Statistical analysis}

Standard descriptive statistics were applied to baseline characteristics of CAD status and coronary revascularization status. Further, we compared the baseline characteristics by BP treatment arms among patients with CAD.

We calculated mean SBP and DBP every 3 months and the mean number of BP-lowering medications every 6 months using all values from the two BP treatment groups during follow-up. These results were summarized in a line graph. Kaplan-Meier survival curves were plotted, and clinical outcomes and safety event rates were computed using Cox proportional hazards regression adjusted for covariates, including demographic characteristics (age and gender), health habits (smoking status), and health condition (body mass index; levels of fasting plasma glucose, lipoprotein cholesterol, and triglycerides; estimated glomerular filtration rate; and use of BP-lowering medications). In addition, we conducted landmark analysis to compare primary outcomes between groups. Furthermore, subgroup analysis was used to assess the effects of CAD status and coronary revascularization status on BP treatment strategies, and these data were presented in a forest plot. All tests were two-sided, and the significance level was set at $P<0.05$. Statistical analyses were performed with SPSS software, version 25 (Chicago, IL, USA) and R 3.6.3 (http://www.R-project.org).

\section{Results}

\section{Study cohort and population characteristics}

Of the 9361 participants in the SPRINT study, there were 1206 participants with CAD (of whom 692 underwent coronary revascularization) and 8127 participants without $\mathrm{CAD}$ 
Table 1 Baseline characteristics of CAD participants by BP treatment arm.

\begin{tabular}{|c|c|c|c|c|}
\hline Characteristics & Total & Standard BP treatment & Intensive $\mathrm{BP}$ treatment & $P$ value \\
\hline$N$ & 1206 & 584 & 622 & \\
\hline Age, years & $70.0 \pm 9.1$ & $69.7 \pm 9.3$ & $70.1 \pm 9.0$ & 0.38 \\
\hline Female, $n(\%)$ & $246(20.4)$ & $132(22.6)$ & $114(18.3)$ & 0.07 \\
\hline Black race, $n(\%)$ & $220(18.2)$ & $113(19.3)$ & $107(17.2)$ & 0.34 \\
\hline Body mass index, $\mathrm{kg} / \mathrm{m}^{2}$ & $29.5 \pm 5.4$ & $29.7 \pm 5.4$ & $29.4 \pm 5.3$ & 0.38 \\
\hline Systolic blood pressure, $\mathrm{mm} \mathrm{Hg}$ & $137.8 \pm 15.7$ & $136.9 \pm 16.2$ & $138.6 \pm 15.5$ & 0.07 \\
\hline Diastolic blood pressure, $\mathrm{mm} \mathrm{Hg}$ & $74.1 \pm 12.1$ & $73.6 \pm 12.0$ & $74.7 \pm 12.1$ & 0.14 \\
\hline Heart rate, bpm & $62.7 \pm 11.1$ & $63.2 \pm 11.7$ & $62.6 \pm 10.7$ & 0.34 \\
\hline Chronic kidney disease, $n(\%)$ & $442(36.7)$ & $216(37.0)$ & $226(36.3)$ & 0.81 \\
\hline Smoking status, $n(\%)$ & & & & 0.67 \\
\hline Never smoked & $401(33.3)$ & $188(32.2)$ & $213(34.2)$ & \\
\hline Former smoker & $647(53.6)$ & $321(55.0)$ & $326(52.4)$ & \\
\hline Current smoker & $158(13.1)$ & $75(12.8)$ & $83(13.3)$ & \\
\hline Total cholesterol, mg/dl & $165.9 \pm 39.2$ & $167.4 \pm 40.0$ & $165.5 \pm 40.2$ & 0.42 \\
\hline LDL-C, mg/dl & $92.4 \pm 33.7$ & $92.8 \pm 33.2$ & $91.8 \pm 34.1$ & 0.62 \\
\hline HDL-C, mg/dl & $48.8 \pm 12.2$ & $48.7 \pm 12.3$ & $48.9 \pm 12.3$ & 0.71 \\
\hline Triglycerides, mg/dl & $123.8 \pm 62.3$ & $128.9 \pm 75.4$ & $126.5 \pm 91.8$ & 0.61 \\
\hline Fasting plasma glucose, $\mathrm{mg} / \mathrm{dl}$ & $100.5 \pm 13.5$ & $100.1 \pm 13.0$ & $100.9 \pm 14.0$ & 0.27 \\
\hline $\mathrm{eGFR}, \mathrm{mL} / \mathrm{min} / 1.73 \mathrm{~m}^{2}$ & $67.2 \pm 19.8$ & $67.1 \pm 19.7$ & $67.8 \pm 20.0$ & 0.55 \\
\hline Creatinine, $\mathrm{mg} / \mathrm{dl}$ & $1.2 \pm 0.3$ & $1.1 \pm 0.3$ & $1.1 \pm 0.4$ & 0.95 \\
\hline Serum sodium, mmol/l & $140.2 \pm 2.5$ & $140.1 \pm 2.5$ & $140.2 \pm 2.6$ & 0.64 \\
\hline Serum potassium, $\mathrm{mmol} / \mathrm{l}$ & $4.3 \pm 0.4$ & $4.3 \pm 0.4$ & $4.3 \pm 0.4$ & 0.08 \\
\hline Statin use, $n(\%)$ & $946(78.4)$ & $457(78.3)$ & 489 (79.6) & 0.56 \\
\hline Aspirin use, $n(\%)$ & $1027(85.2)$ & $488(83.6)$ & $539(86.7)$ & 0.13 \\
\hline Antihypertensive agents, $n(\%)$ & & & & 0.34 \\
\hline 1 & $246(20.4)$ & $111(19.0)$ & $135(21.7)$ & \\
\hline 2 & $472(39.1)$ & $227(38.9)$ & $245(39.4)$ & \\
\hline 3 & $333(27.6)$ & $171(29.3)$ & $162(26.0)$ & \\
\hline 4 & $97(8.0)$ & $47(8.0)$ & $50(8.0)$ & \\
\hline
\end{tabular}

Values are mean \pm SD or number (\%).

$C A D$ coronary artery disease, $L D L-C$ low-density lipoprotein cholesterol, $H D L-C$ high-density lipoprotein cholesterol, $e G F R$ estimated glomerular filtration rate. at baseline (Supplementary Fig. 1). There were significant differences in baseline characteristics between participants with and without CAD (Supplementary Table 1). The baseline characteristics of participants with and without coronary revascularization are shown in Supplementary Table 2.

The baseline characteristics of participants with CAD were comparable $(P>0.05)$ between the standard and intensive groups (Table 1). At baseline, the mean BP was $136.9 \pm 16.2 / 73.6 \pm 12.0 \mathrm{~mm} \mathrm{Hg}$ in the standard group and $138.6 \pm 15.5 / 74.7 \pm 12.1 \mathrm{~mm} \mathrm{Hg}$ in the intensive group. In both groups, the mean BP levels were controlled to within the target range $(133.7 \pm 3.0 / 71.0 \pm 1.4 \mathrm{~mm} \mathrm{Hg}$ vs. $120.9 \pm$ $2.5 / 64.6 \pm 1.6 \mathrm{~mm} \mathrm{Hg}$ ) during follow-up. The mean number of BP-lowering medications in the standard and intensive groups was 2.1 and 3.0, respectively (Fig. 1). The BPlowering medications of different groups are summarized in Supplementary Tables 3 and 4.

\section{Subgroup analysis by CAD status}

After a median follow-up of 3.9 years (interquartile range 3.4-4.4 years), primary composite events were documented in 150 participants with CAD (70 in the standard group and 80 in the intensive group) (Table 2). Intensive $\mathrm{BP}$ treatment was associated with a lower risk of all-cause death (adjusted hazard ratio [HR] 0.60, 95\% confidence interval [CI] 0.37-0.96) and a trend for an increased risk of stroke (adjusted HR 2.08, 95\% CI 0.94-4.58), compared with standard BP treatment (Fig. 2A, B). The primary outcome, as well as most secondary outcomes, were similar in the two groups (Table 2, Fig. 2C). The landmark analysis showed that the HRs for the primary outcome were 1.10 (95\% CI 0.79-1.54) and 0.57 (95\% CI $0.18-1.79$ ) within and after the first 3.4 years, respectively (Fig. 2D). 


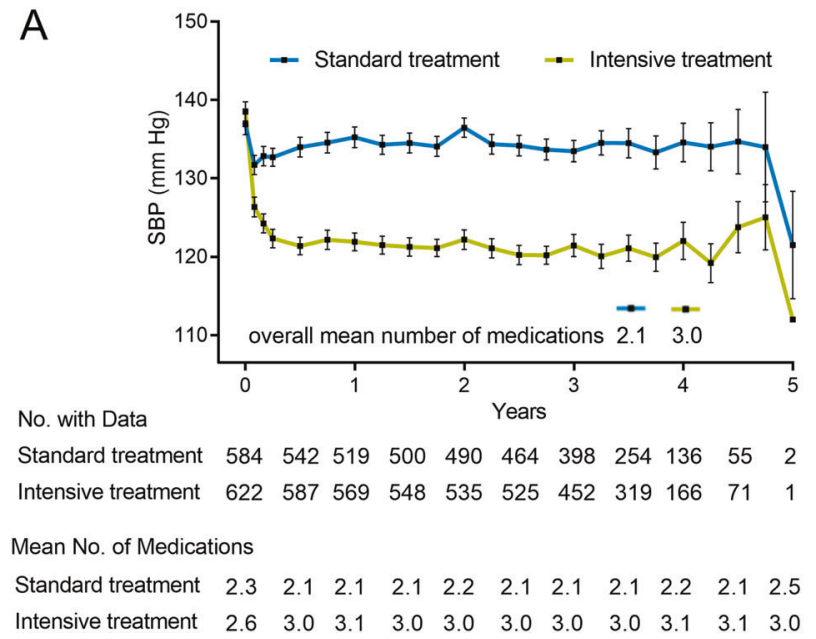

Fig. 1 Blood pressure for participants with CAD through the 5-years follow-up visit. Mean follow-up SBP (A) and DBP (B) in the two BP treatment groups in participants with CAD. Mean number of
B

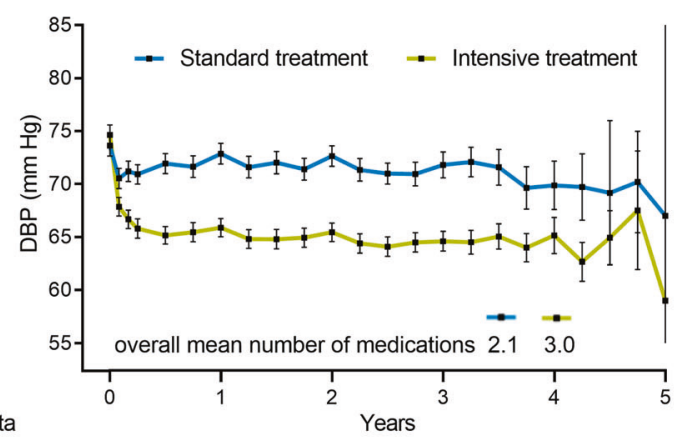

No. with Data

$\begin{array}{llllllllllll}\text { Standard treatment } & 584 & 542 & 519 & 500 & 490 & 464 & 398 & 254 & 136 & 55 & 2\end{array}$ Intensive treatment $\quad \begin{array}{lllllllllll}622 & 587 & 569 & 548 & 535 & 525 & 452 & 319 & 166 & 71 & 1\end{array}$

\section{Mean No. of Medications}

$\begin{array}{llllllllllll}\text { Standard treatment } & 2.3 & 2.1 & 2.1 & 2.1 & 2.2 & 2.1 & 2.1 & 2.1 & 2.2 & 2.1 & 2.5\end{array}$

$\begin{array}{llllllllllll}\text { Intensive treatment } & 2.6 & 3.0 & 3.1 & 3.0 & 3.0 & 3.0 & 3.0 & 3.0 & 3.1 & 3.1 & 3.0\end{array}$

BP-lowering medications are noted every 6 months during follow-up. Bars represent $95 \%$ CI. SBP systolic blood pressure, DBP diastolic blood pressure, CAD coronary artery disease, CI confidence interval.

Table 2 Primary and second outcomes in CAD participants by BP treatment arm.

\begin{tabular}{|c|c|c|c|c|c|c|}
\hline \multirow[t]{2}{*}{ Outcome } & \multirow[t]{2}{*}{ Standard BP treatment } & \multirow[t]{2}{*}{ Intensive $\mathrm{BP}$ treatment } & \multicolumn{2}{|l|}{ Unadjusted model } & \multicolumn{2}{|l|}{ Adjusted model $^{\mathrm{a}}$} \\
\hline & & & HR $(95 \% \mathrm{CI})$ & $P$ value & HR $(95 \% \mathrm{CI})$ & $P$ value \\
\hline$N$ & 584 & 622 & & & & \\
\hline Primary outcome & $70(12.0)$ & $80(12.9)$ & $1.04(0.76-1.44)$ & 0.80 & $1.05(0.76-1.46)$ & 0.75 \\
\hline \multicolumn{7}{|l|}{ Secondary outcomes } \\
\hline Myocardial infarction & $28(4.8)$ & $32(5.1)$ & $1.03(0.62-1.72)$ & 0.90 & $1.05(0.62-1.75)$ & 0.87 \\
\hline ACS & $16(2.7)$ & $21(3.4)$ & $1.20(0.63-2.31)$ & 0.58 & $1.22(0.64-2.35)$ & 0.55 \\
\hline Stroke & $9(1.5)$ & $20(3.2)$ & $2.03(0.93-4.46)$ & 0.08 & $2.08(0.94-4.58)$ & 0.07 \\
\hline Heart failure & $23(3.9)$ & $16(2.6)$ & $0.62(0.33-1.18)$ & 0.15 & $0.61(0.32-1.17)$ & 0.14 \\
\hline CVD death & $15(2.6)$ & $13(2.1)$ & $0.77(0.37-1.62)$ & 0.49 & $0.75(0.35-1.63)$ & 0.47 \\
\hline All-cause death & $45(7.7)$ & $31(5.0)$ & $0.62(0.39-0.98)$ & 0.04 & $0.60(0.37-0.96)$ & 0.03 \\
\hline
\end{tabular}

Values are presented as number $(\%)$ or HR $(95 \% \mathrm{CI})$

$C A D$ coronary artery disease, ACS acute coronary syndrome, $C V D$ cardiovascular disease, $H R$ hazard ratio, $C I$ confidence interval.

adjusted model: age, gender, smoking status, body mass index, triglycerides, low-density lipoprotein cholesterol, high-density lipoprotein cholesterol, fasting plasma glucose, estimated glomerular filtration rate, and use of antihypertensive agents.

The SPRINT study showed that intensive BP treatment resulted in a low rate of major cardiovascular events, but we found no benefits on the primary outcome in participants with pre-existing CAD. Therefore, we further conducted subgroup analysis to verify whether CAD status plays a key role in the effects of BP treatment on clinical outcomes (Fig. 3). The results showed that the HRs for the primary outcome were 0.65 (95\% CI $0.53-0.79)$ and 1.05 (95\% CI $0.76-1.46$ ) among those in the non-CAD and CAD subgroup, respectively ( $P$ value for interaction 0.02 ). For participants without $\mathrm{CAD}$, intensive $\mathrm{BP}$ treatment significantly decreased the risk of myocardial infarction, heart failure, CVD death, and allcause death, compared with standard BP treatment.

\section{Subgroup analysis by coronary revascularization status}

In addition, participants with CAD were categorized by coronary revascularization status. The HRs for primary outcome were 1.27 (95\% CI 0.82-1.95) and 0.82 (95\% CI 0.49-1.37) in CAD participants with and without coronary revascularization, respectively ( $P$ value for interaction 0.24). Intensive BP treatment appeared to reduce the risk of all-cause death (HR $0.47,95 \%$ CI $0.22-1.01$ ) in participants without coronary revascularization, and there was no increase in the risk of stroke (HR $1.03,95 \% \mathrm{CI}$ 0.29-3.62). However, in the subgroup of participants with 
A

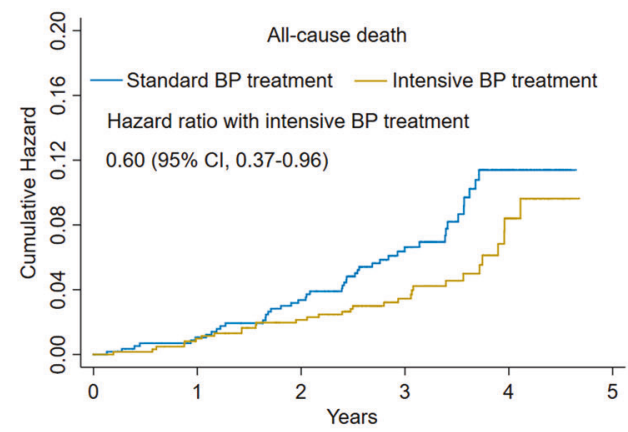

Number at risk

$\begin{array}{llllll}\text { Standard BP treatment } 576 & 563 & 540 & 334 & 91 & 0 \\ \text { Intensive BP treatment } 619 & 604 & 585 & 387 & 97 & 0\end{array}$

C

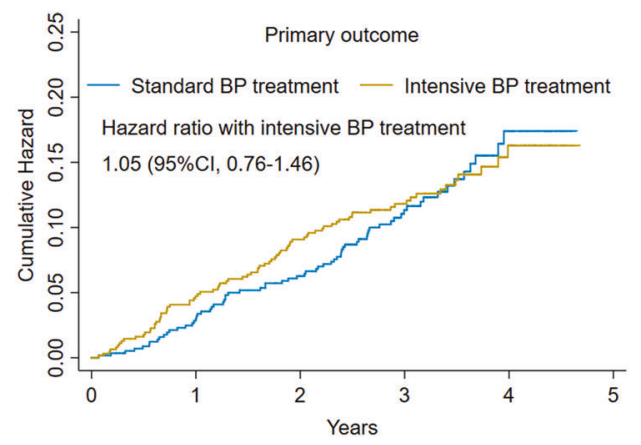

Number at risk

Standard BP treatment 572 Intensive BP treatment 618 544
579 $544 \quad 511$ 511
538 300
347 72
87

Fig. 2 Cumulative incidence for clinical outcomes in participants with CAD, comparing treatment assignments. Cumulative incidence of the all-cause death $(\mathbf{A})$, stroke $(\mathbf{B})$, primary outcome $(\mathbf{C})$, and the primary outcome with landmark analysis (D) according to standard
B

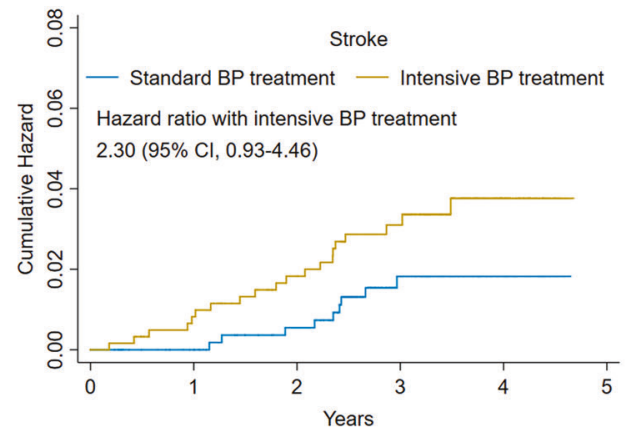

Number at risk

$\begin{array}{llllll}\text { Standard BP treatment } 572 & 556 & 536 & 325 & 89 & 0 \\ \text { Intensive BP treatment 617 } & 599 & 576 & 373 & 93 & 0\end{array}$

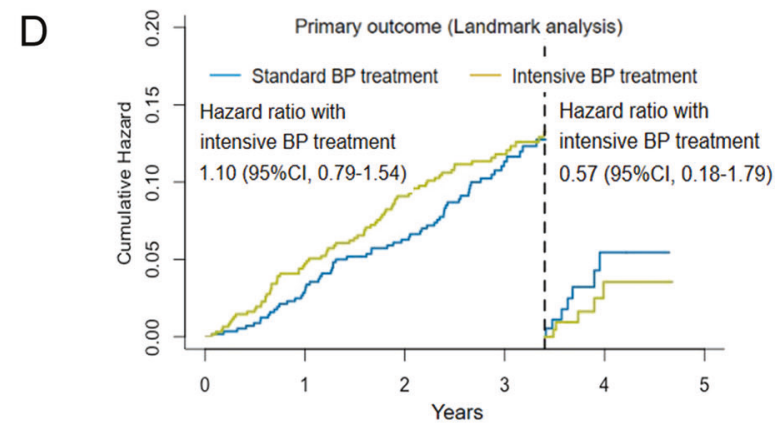

Number at risk

$\begin{array}{llllll}\text { Standard BP treatment } 572 & 544 & 511 & 300 & 72 & 0 \\ \text { Intensive BP treatment 618 } & 579 & 538 & 347 & 87 & 0\end{array}$

and intensive BP treatments in participants with CAD. BP blood pressure, $\mathrm{CAD}$ coronary artery disease, $\mathrm{HR}$ hazard ratio, $\mathrm{CI}$ confidence interval.
Fig. 3 Test for interaction between BP treatment assignment and baseline CAD status. BP blood pressure, CAD coronary artery disease, MI myocardial infarction, ACS acute coronary syndrome, CVD cardiovascular disease, HR hazard ratio, CI confidence interval.

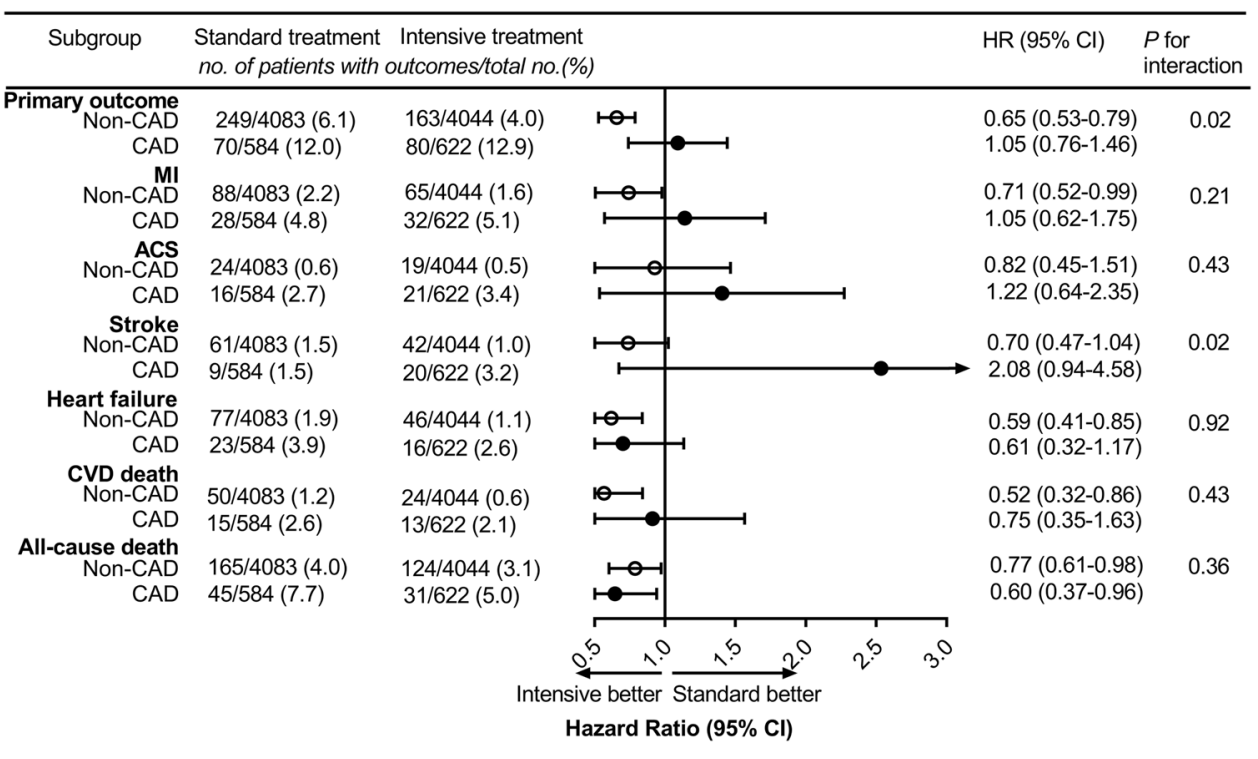

Safety events in participants with CAD

coronary revascularization, intensive $\mathrm{BP}$ treatment had no benefit on all-cause death (HR $0.74,95 \%$ CI $0.40-1.36$ ) but increased the risk of stroke (HR 3.57, 95\% CI 1.17-10.85) (Fig. 4).
Information regarding safety events in participants with CAD is summarized in Table 3 . The incidence of total 
Fig. 4 Test for interaction between BP treatment assignment and baseline coronary revascularization status in CAD participants. BP blood pressure, CAD coronary artery disease, Revas coronary revascularization, MI myocardial infarction, ACS acute coronary syndrome, CVD cardiovascular disease, HR hazard ratio, CI confidence interval.
Table 3 Safety events in CAD participants by BP treatment arm.

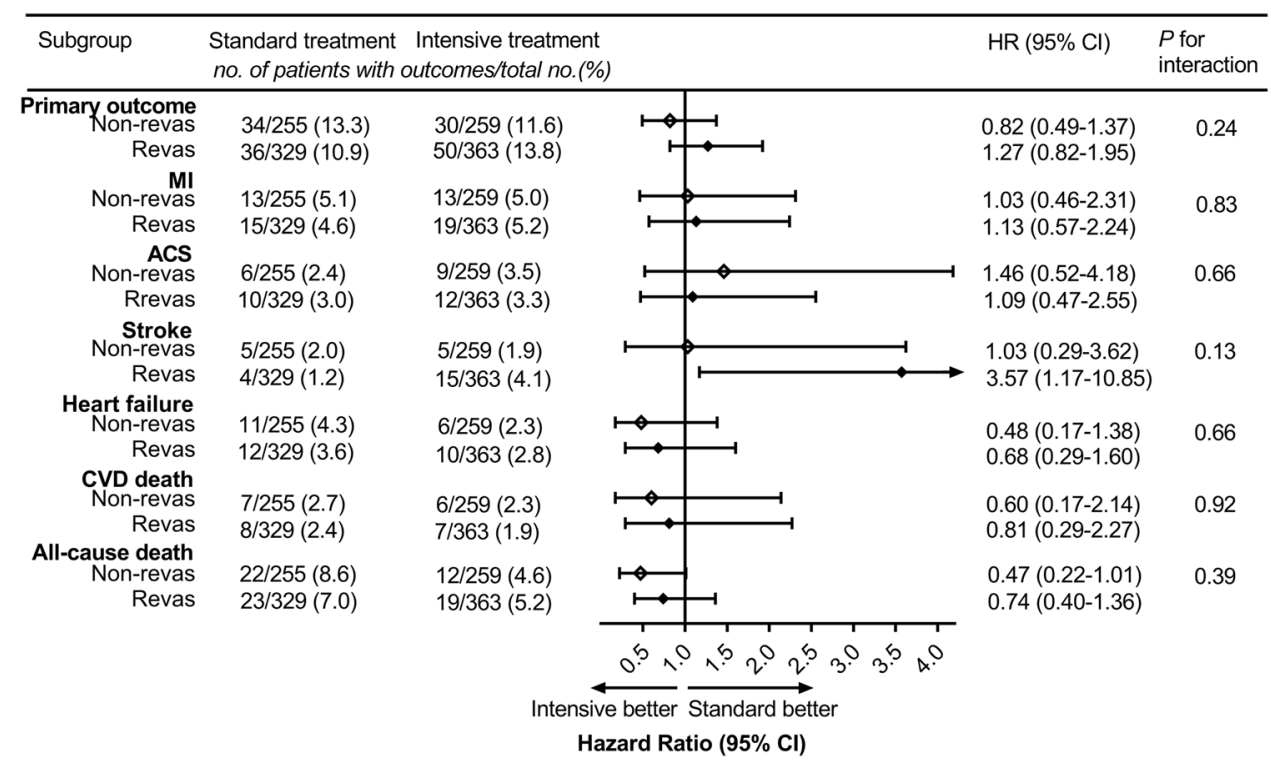

\begin{tabular}{lllll}
\hline Safety events $^{\mathrm{a}}$ & Standard BP treatment & Intensive BP treatment & HR $(95 \%$ CI $)$ & $P$ value \\
\hline$N$ & 584 & 622 & & \\
Hypotension & $14(2.4)$ & $30(4.8)$ & $2.00(1.06-3.79)$ & 0.03 \\
Syncope & $18(3.1)$ & $15(2.4)$ & $0.73(0.37-1.47)$ & 0.38 \\
Electrolyte abnormality & $13(2.2)$ & $33(5.3)$ & $2.38(1.25-4.56)$ & 0.01 \\
Injurious fall & $14(2.4)$ & $19(3.1)$ & $1.21(0.60-2.43)$ & 0.59 \\
Acute kidney injury & $25(4.3)$ & $38(6.1)$ & $1.39(0.82-2.33)$ & 0.22 \\
Bradycardia & $21(3.6)$ & $27(4.3)$ & $1.12(0.63-1.98)$ & 0.71 \\
SAEs & $310(53.1)$ & $340(54.7)$ & $1.03(0.88-1.20)$ & 0.73 \\
\hline
\end{tabular}

Values are presented as number $(\%)$ or HR $(95 \% \mathrm{CI})$.

$C A D$ coronary artery disease, $B P$ blood pressure, $H R$ hazard ratio, $C I$ confidence interval.

${ }^{a}$ Safety events, including hypotension, syncope, electrolyte abnormality, injurious fall, acute kidney injury, and bradycardia.

${ }^{b}$ SAEs indicate serious adverse events, that resulted in significant dysfunction and required medical or hospitalization to surgical intervention.
SAEs in the intensive group $(54.7 \%, 340 / 622)$ was similar to that in the standard group $(53.1 \%, 310 / 584)$. Intensive BP treatment increased the risk of hypotension (HR 2.00, 95\% CI 1.06-3.79) and electrolyte abnormalities (HR 2.38, 95\% CI 1.25-4.56). There was no increased risk of other safety events including syncope, injurious fall, acute kidney injury, and bradycardia in participants with intensive BP treatment, compared with standard BP treatment.

\section{Discussion}

Our results indicate that $\mathrm{CAD}$ and coronary revascularization status influence the effect of BP treatment on clinical outcomes. Intensive BP treatment decreased the risk of major cardiovascular events in participants without CAD, but not in those with CAD. For CAD participants, intensive
BP treatment is associated with a reduced risk for all-cause death but does not affect other clinical outcomes, compared with standard BP treatment. The cardiovascular benefits from intensive BP treatment were further attenuated and the risk of stroke might increase in patients with CAD and a history of coronary revascularization.

The results of this study add to the complex evidence for the interaction and association of $\mathrm{BP}$ control with $\mathrm{CAD}$ status and coronary revascularization. We found a lower risk of clinical outcomes with an SBP target of $120 \mathrm{~mm} \mathrm{Hg}$ among hypertensive patients without CAD. Attar et al. performed a further analysis of SPRINT participants categorized based on the baseline 10-year Framingham risk, and their results indicated that intensive BP treatment is beneficial for primary prevention of CVD and mortality in patients with high risk (above 10\%) [15]. In contrast, another clinical trial (ONgoing Telmisartan Alone and in 
combination with Ramipril Global Endpoint Trial, ONTARGET) found that reducing SBP below $130 \mathrm{~mm} \mathrm{Hg}$ failed to reduce cardiovascular mortality and that the optimal SBP was $135-145 \mathrm{~mm} \mathrm{Hg}$ in patients with high cardiovascular risk [16].

The optimal BP targets in hypertensive patients with $\mathrm{CAD}$ remain controversial as few randomized clinical trials have directly evaluated this important clinical question. There are several existing studies nested with CAD populations, but these did not examine specific SBP targets. The INternational VErapamil SR Trandolapril STudy (INVEST) conducted by Pepine et al. evaluated 22,576 hypertensive CAD patients aged 50 years or older and found the verapamil-trandolapril-based strategy was as clinically effective as the atenolol-hydrochlorothiazide-based strategy, suggesting that reducing SBP is a far more important clinical consideration than the choice of antihypertensive drug class [17]. A post-hoc analysis of INVEST evaluated 8354 participants $\geq 60$ years of age with hypertension and CAD and concluded that these patients may benefit from reducing their SBP below $140 \mathrm{~mm} \mathrm{Hg}$ [18]. Similar patterns were also observed in the PRavastatin Or atorVastatin Evaluation and Infection Therapy-Thrombolysis In Myocardial Infarction (PROVE IT-TIMI) 22 trial, which found that the lowest event rates were associated with an SBP range of 130-140 $\mathrm{mm} \mathrm{Hg}$ in patients with acute coronary syndrome. The Comparison of Amlodipine vs Enalapril to Limit Occurrences of Thrombosis study conducted by Nissen et al. included $1991 \mathrm{CAD}$ patients with an average baseline BP of $129 / 78 \mathrm{~mm} \mathrm{Hg}$ and demonstrated that treatment with amlodipine resulted in reduced adverse cardiovascular events in normotensive patients with CAD [19]. The above studies suggest that the optimal SBP level in patients with CAD is clearly lower than $140 \mathrm{~mm} \mathrm{Hg}$ and perhaps in 120 $\mathrm{mm} \mathrm{Hg}$ range [20]. According to the recent findings of a network meta-analysis, the 2017 American College of Cardiology/American Heart Association practice guideline updated its recommendation for an SBP target to $<130 \mathrm{~mm}$ $\mathrm{Hg}$ in adults with SIHD [8, 14, 21].

Because coronary perfusion occurs mainly during diastole, DBP has gradually become the focus of attention in hypertensive patients with CAD. A secondary analysis using data obtained from the INVEST observed a J-shaped association between BP and cardiovascular events, in which the J-curve was relatively more prominent in diastole than in systole. Moreover, the authors found that patients who had coronary revascularization seemed to have the potential to tolerate the lower DBP, compared with those who had not $[13,17]$. The current BP management guideline recommends a target DBP of $<80 \mathrm{~mm} \mathrm{Hg}$ in patients with SIHD [8]. In addition, the PROVE IT-TIMI 22 trial suggested a $\mathrm{DBP}<70 \mathrm{~mm} \mathrm{Hg}$ may be dangerous in patients with high-risk unstable angina [22]. Furthermore, the
Treating to New Targets Trial revealed that a low DBP of less than $60-70 \mathrm{~mm} \mathrm{Hg}$ was accompanied by an increased risk of cardiovascular events in patients with CAD [23]. Similar findings were observed in the Atherosclerosis Risk in Communities study [24]. Therefore, DBP should be considered during hypertension treatment [25]. Unlike the secondary analysis of the previous studies, our results showed that a DBP around $65 \mathrm{~mm} \mathrm{Hg}$ in patients with CAD was still safe and did not increase CVD events.

In addition, we found that participants with a history of coronary revascularization had lower DBP regardless of antihypertensive treatment, compared to those without such a history. The most probable explanation is that individuals with revascularization have worse atherosclerotic lesions and poorer arterial elasticity. The loss of arterial elasticity results in a decline in DBP and impairment of the auto-regulatory process of coronary circulation [26, 27]. Although revascularization opens culprit vessels and restores blood flow to ischemic areas, such a procedure per se cannot prevent atherosclerotic progression. On the one hand, it is not recommended to achieve an SBP target of $120 \mathrm{~mm} \mathrm{Hg}$ in CAD patients with coronary revascularization, as our results suggest that this may increase the risk of stroke. On the other hand, excessive diastolic hypotension may have attenuated the benefits from intensive SBP treatment [28]. Further research is required to evaluate the minimum DBP target that has no effect on myocardial perfusion while ensuring adequate reduction in SBP in CAD patients with coronary revascularization.

We analyzed the baseline characteristics of participants with and without CAD or coronary revascularization. We observed that traditional cardiovascular risk factors, including heart rate, total cholesterol, low-density lipoprotein cholesterol, and use of aspirin and statins, were better controlled in participants who had CAD or had undergone coronary revascularization than in those who had not. In light of this, we postulate that the benefits of further intensive BP treatment might have been diluted in this population due to existing well-executed secondary prevention. It is, however, undeniable that the relatively limited subject number reduced the power of the study.

Several limitations of the present study should be noted. First, it was based on a post-hoc analysis of data obtained from a randomized controlled trial. The number of participants with CAD was relatively small, which may reduce the power of our statistical analysis. Second, the SPRINT study population excluded patients with a history of diabetes mellitus or stroke; thus, our study conclusions may not apply to other subsets of patients. Third, BP measurements in an unattended office are not an alternative to home BP measurements, given the low correlation and wide range of differences between the two groups [29]. Therefore, these 
results should be carefully interpreted and further validated through future studies.

In conclusion, the present study suggested that patients without CAD whose SBP is around $120 \mathrm{~mm} \mathrm{Hg}$ have greater benefits for clinical outcomes, while this benefits might be attenuated in patients with CAD who are under better secondary prevention. The risk of stroke was increased by intensive BP treatment in patients with a history of coronary revascularization, though the confidence interval was wide. These findings may serve to inform current clinical practice and future trial design.

\section{Summary}

\section{What is known about the topic}

- The prevalence of hypertension in individuals with preexisting CAD ranges from 30 to $70 \%$.

- Uncontrolled BP increased mortality in patients with CAD.

- Recent practice guidelines recommend a BP target of less than 130/80 $\mathrm{mm} \mathrm{Hg}$ in individuals with SIHD.

\section{What this study adds}

- The optimal SBP level in hypertensive patients without CAD and diabetes mellitus is around $120 \mathrm{~mm} \mathrm{Hg}$.

- In non-diabetic hypertensive patients with CAD who are under better secondary prevention, the benefit from intensive BP treatment might be attenuated.

- The risk of stroke is increased by an SBP target of 120 $\mathrm{mm} \mathrm{Hg}$ in patients with a history of coronary revascularization, though the confidence interval was wide.

Funding This study was founded by Shenzhen Key Medical Discipline Construction Fund (No: SZXK002), National Natural Science Foundation of China (No: 81970367, 81670417 to GW), Natural Science Foundation of Guangdong Province (No: 2018A030313807 to JL), the Science and Technology Planning Project of Shenzhen Municipality (No: JCYJ20180306180229307 to JL), and Sun Yat-sen University Clinical Medicine Research 5010 Cultivation Project (No: 2018027 to JL).

Author contributions (I) Conception and design: GW, XL, JZ; (II) Administrative support: GW; (III) Provision of study materials or patients: XZ, JL; (IV) Collection and assembly of data: JZ, JL, SZ; (V) Data analysis and interpretation: JZ, XZ; (VI) Manuscript writing: All authors; (VII) Final approval of manuscript: All authors.

\section{Compliance with ethical standards}

Conflict of interest The authors declare no competing interests.
Ethical approval The study protocol was reviewed and approved by the Ethics Committee of the First Affiliated Hospital of Sun Yat-sen University.

Publisher's note Springer Nature remains neutral with regard to jurisdictional claims in published maps and institutional affiliations.

Open Access This article is licensed under a Creative Commons Attribution 4.0 International License, which permits use, sharing, adaptation, distribution and reproduction in any medium or format, as long as you give appropriate credit to the original author(s) and the source, provide a link to the Creative Commons license, and indicate if changes were made. The images or other third party material in this article are included in the article's Creative Commons license, unless indicated otherwise in a credit line to the material. If material is not included in the article's Creative Commons license and your intended use is not permitted by statutory regulation or exceeds the permitted use, you will need to obtain permission directly from the copyright holder. To view a copy of this license, visit http://creativecommons. org/licenses/by/4.0/.

\section{References}

1. Stamler J, Stamler R, Neaton JD. Blood pressure, systolic and diastolic, and cardiovascular risks. Us population data. Arch Intern Med. 1993;153:598-615.

2. Rapsomaniki E, Timmis A, George J, Pujades-Rodriguez M, Shah $\mathrm{AD}$, Denaxas S, et al. Blood pressure and incidence of twelve cardiovascular diseases: lifetime risks, healthy life-years lost, and age-specific associations in 1.25 million people. Lancet. 2014;383:1899-911.

3. Forouzanfar MH, Liu P, Roth GA, Ng M, Biryukov S, Marczak L, et al. Global burden of hypertension and systolic blood pressure of at least 110 to $115 \mathrm{~mm} \mathrm{Hg}, 1990-2015$. JAMA. 2017;317:165-82.

4. Lewington S, Lacey B, Clarke R, Guo Y, Kong XL, Yang L, et al. The burden of hypertension and associated risk for cardiovascular mortality in china. JAMA Intern Med. 2016;176:524-32.

5. Mahtta D, Elgendy IY, Pepine CJ. Optimal medical treatment of hypertension in patients with coronary artery disease. Expert Rev Cardiovasc Ther. 2018;16:815-23.

6. Lewington S, Clarke R, Qizilbash N, Peto R, Collins R, Prospective Studies C, Prospective Studies Collaboration. Agespecific relevance of usual blood pressure to vascular mortality: A meta-analysis of individual data for one million adults in 61 prospective studies. Lancet. 2002;360:1903-13.

7. Oparil S, Acelajado MC, Bakris GL, Berlowitz DR, Cifkova R, Dominiczak AF, et al. Hypertension. Nat Rev Dis Prim. 2018;4:18014.

8. Whelton PK, Carey RM, Aronow WS, Casey DE Jr, Collins KJ, Dennison Himmelfarb C, et al. 2017 ACC/AHA/AAPA/ABC/ ACPM/AGS/APHA/ASH/ASPC/NMA/PCNA guideline for the prevention, detection, evaluation, and management of high blood pressure in adults. Hypertension. 2018;71:e13-e115.

9. Cruickshank JM. Coronary flow reserve and the $\mathrm{j}$ curve relation between diastolic blood pressure and myocardial infarction. BMJ. 1998;297:1227-30.

10. Rahman F, McEvoy JW. The j-shaped curve for blood pressure and cardiovascular disease risk: historical context and recent updates. Curr Atheroscler Rep. 2017;19:34.

11. Kjeldsen SE, Berge E, Bangalore S, Messerli FH, Mancia G, Holzhauer B, et al. No evidence for a j-shaped curve in treated hypertensive patients with increased cardiovascular risk: The VALUE trial. Blood Press. 2016;25:83-92. 
12. Di Nora C, Cioffi G, Iorio A, Rivetti L, Poli S, Zambon E, et al. Systolic blood pressure target in systemic arterial hypertension: Is lower ever better? Results from a community-based caucasian cohort. Eur J Intern Med. 2018;48:57-63.

13. Messerli FH, Mancia G, Conti CR, Hewkin AC, Kupfer S, Champion A, et al. Dogma disputed: can aggressively lowering blood pressure in hypertensive patients with coronary artery disease be dangerous? Ann Intern Med. 2006;144:884-93.

14. SPRINT Research Group, Wright JT Jr, Williamson JD, Whelton PK, Snyder JK, Sink KM, et al. A randomized trial of intensive versus standard blood-pressure control. N. Engl J Med. 2015;373:2103-16.

15. Attar A, Sayadi M, Jannati M. Effect of intensive blood pressure lowering on cardiovascular outcomes based on cardiovascular risk: a secondary analysis of the SPRINT trial. Eur J Prev Cardiol. 2019;26:238-45.

16. Sleight P, Redon J, Verdecchia P, Mancia G, Gao P, Fagard R, et al. Prognostic value of blood pressure in patients with high vascular risk in the ongoing telmisartan alone and in combination with ramipril global endpoint trial study. J Hypertens. 2009;27:1360-9.

17. Pepine CJ, Handberg EM, Cooper-DeHoff RM, Marks RG, Kowey $\mathrm{P}$, Messerli FH, et al. A calcium antagonist vs a noncalcium antagonist hypertension treatment strategy for patients with coronary artery disease. The international verapamiltrandolapril study (INVEST): a randomized controlled trial. JAMA. 2003;290:2805-16.

18. Bangalore S, Gong Y, Cooper-DeHoff RM, Pepine CJ, Messerli FH. 2014 Eighth Joint National Committee panel recommendation for blood pressure targets revisited: results from the INVEST study. J Am Coll Cardiol. 2014;64:784-93.

19. Nissen SE, Tuzcu EM, Libby P, Thompson PD, Ghali M, Garza $\mathrm{D}$, et al. Effect of antihypertensive agents on cardiovascular events in patients with coronary disease and normal blood pressure the CAMELOT study: a randomized controlled trial. JAMA. 2004;292:2217-25.

20. Pepine CJ. What is the optimal blood pressure and drug therapy for patients with coronary artery disease? JAMA. 2004;292:2271-3.
21. Bundy JD, Li C, Stuchlik P, Bu X, Kelly TN, Mills KT, et al. Systolic blood pressure reduction and risk of cardiovascular disease and mortality: a systematic review and network metaanalysis. JAMA Cardiol. 2017;2:775-81.

22. Bangalore S, Qin J, Sloan S, Murphy SA, Cannon CP, PROVE IT-TIMI 22 Trial Investigators. What is the optimal blood pressure in patients after acute coronary syndromes?: Relationship of blood pressure and cardiovascular events in the pravastatin or atorvastatin evaluation and infection therapy-thrombolysis in myocardial infarction (PROVE IT-TIMI) 22 trial. Circulation. 2010;122:2142-51.

23. Bangalore S, Messerli FH, Wun CC, Zuckerman AL, DeMicco D, Kostis JB, et al. J-curve revisited: An analysis of blood pressure and cardiovascular events in the treating to new targets (TNT) trial. Eur Heart J. 2010;31:2897-908.

24. McEvoy JW, Chen Y, Rawlings A, Hoogeveen RC, Ballantyne $\mathrm{CM}$, Blumenthal RS, et al. Diastolic blood pressure, subclinical myocardial damage, and cardiac events: implications for blood pressure control. J Am Coll Cardiol. 2016;68:1713-22.

25. Böhm M, Schumacher H, Teo KK, Lonn EM, Mahfoud F, Mann JFE, et al. Achieved blood pressure and cardiovascular outcomes in high-risk patients: results from ONTARGET and TRANSCEND trials. Lancet. 2018;389:2226-37.

26. Witteman JC, Grobbee DE, Valkenburg HA, van Hemert AM, Stijnen T, Burger $\mathrm{H}$, et al. J-shaped relation between change in diastolic blood pressure and progression of aortic atherosclerosis. Lancet. 1994;343:504-7.

27. Casadonte L, Verhoeff BJ, Piek JJ, VanBavel E, Spaan JAE, Siebes M. Influence of increased heart rate and aortic pressure on resting indices of functional coronary stenosis severity. Basic Res Cardiol. 2017;112:61.

28. Lee TC, Cavalcanti RB, McDonald EG, Pilote L, Brophy JM. Diastolic hypotension may attenuate benefits from intensive systolic targets: secondary analysis of a randomized controlled trial. Am J Med. 2018;131:1228-33.e1.

29. Asayama K, Ohkubo T, Rakugi H, Miyakawa M, Mori H, Katsuya $\mathrm{T}$, et al. Comparison of blood pressure values-self-measured at home, measured at an unattended office, and measured at a conventional attended office. Hypertens Res. 2019;42:1726-37. 